\title{
ANÁLISE ESPACIAL DO RISCO DE ADOECIMENTO DA HANSENÍASE EM UM ESTADO DO NORDESTE BRASILEIRO
}

\author{
SPATIAL ANALYSIS OF THE RISK OF LEPROSY DISEASE \\ IN A NORTHEASTERN BRAZILIAN STATE
}

\section{ANÁLISIS ESPACIAL DEL RIESGO DE ENFERMEDAD DE LA LEPRA EN UN ESTADO DEL NORESTE BRASILEÑO}

\author{
Kleane Maria da Fonseca Azevedo Araújo ${ }^{1}$ \\ Lidiane Cristina Félix Gomes ${ }^{2}$ \\ Francisco Carlos Félix Lana ${ }^{3}$
}

\begin{abstract}
Como citar este artigo: Araújo KMFA, Gomes LCF, Lana FCF. Análise espacial do risco de adoecimento da hanseníase em um estado do nordeste brasileiro. Rev baiana enferm. 2020;34:e37902.

Objetivo: analisar a distribuição espacial do risco para o adoecimento de hanseníase nos municípios do estado da Paraíba, Brasil. Método: trata-se de estudo ecológico de análise espacial. Foram incluídos casos novos de hanseníase residentes na Paraíba, registrados no Sistema de Informação de Agravos de Notificação de 2001 a 2016. Foi realizada análise espacial descritiva nos períodos de 2001-2008 e 2009-2016 do índice composto de indicadores epidemiológico e análise de dependência espacial por meio dos índices de Moran global e local. Resultados: foram registrados 12.134 casos novos no período estudado. Identificou-se no primeiro período clusters com classificação alto/alto nas regiões leste, oeste e noroeste. No segundo período, os clusters concentram-se nas regiões leste e sudeste e decresceu no oeste e noroeste. Conclusão: o risco para o adoecimento de hanseníase está presente nos municípios da Paraíba. A distribuição espacial é dessemelhante no estado. Ações de vigilância devem ser prioritárias para o controle da endemia.
\end{abstract}

Descritores: Hanseníase. Epidemiologia. Análise Espacial. Atenção Primária à Saúde.

Objective: to analyze the spatial distribution of the risk for leprosy disease in the municipalities of the state of Paraiba, Brazil. Method: this is an ecological study of spatial analysis. New cases of leprosy living in Paraiba, registered in the Notifiable Diseases Information System from 2001 to 2016, were included. Descriptive spatial analysis was performed in the periods of 2001-2008 and 2009-2016 of the composite index of epidemiological indicators and spatial dependence analysis through the global and local Moran indices. Results: 12,134 new cases were recorded in the period studied. Clusters with high/high classification in the east, west and northwest regions were identified in the first period. In the second period, clusters are concentrated in the east and southeast regions and decreased in the west and northwest. Conclusion: the risk for leprosy disease is present in the municipalities of Paraiba. Spatial distribution is dissimilar in the state. Surveillance actions should be a priority for the control of the endemic disease.

Descriptors: Leprosy. Epidemiology. Spatial analysis. Primary Health Care.

Objetivo: analizar la distribución espacial del riesgo de enfermedad de la lepra en los municipios del estado de Paraíba, Brasil. Método: este es un estudio ecológico del análisis espacial. Se incluyeron nuevos casos de lepra que viven en Paraíba, inscritos en el Sistema de Información sobre Enfermedades Notificabbles de 2001 a 2016. El

Enfermeira. Doutora em Enfermagem. Professora na Universidade Federal de Campina Grande. Campina Grande, Paraíba, Brasil. kleanemaria@yahoo.com.br. http:// orcid.org/0000-0001-8033-7305.

${ }^{2}$ Graduação em Tecnologia em Geoprocessamento. Licenciatura em Geografia. Doutorado em Geografia. Professora no Instituto Federal de Educação, Ciência e Tecnologia da Paraíba. Picuí, Paraíba, Brasil. http://orcid.org/0000-000 I-52 I5-6208.

${ }^{3}$ Enfermeiro e Obstetra. Doutor em Enfermagem em Interunidades. Professor Titular na Universidade Federal de Minas Gerais. Belo Horizonte, Minas Gerais, Brasil. http://orcid.org/0000-000 I-9043-3181. 
análisis espacial descriptivo se realizó en los períodos 2001-2008 y 2009-2016 del índice compuesto de indicadores epidemiológicos y análisis de dependencia espacial a través de los indices globales y locales de Moran. Resultados: se registraron 12.134 nuevos casos en el período estudiado. En el primer período se identificaron los clusters con clasificación alta/alta en las regiones este, oeste y noroeste. En el segundo periodo, los clusters se concentran en las regiones este y sureste y disminuyen en el oeste y noroeste. Conclusión: el riesgo de enfermedad de la lepra está presente en los municipios de Paraíba. La distribución espacial es diferente en el estado. Las acciones de vigilancia deben ser una prioridad para el control de la enfermedad endémica.

Descriptores: Lepra. Epidemiología. Análisis espacial. Atención Primaria de Salud.

\section{Introdução}

A hanseníase é uma doença transmissível, de evolução crônica, que causa deformidades e incapacidades se não diagnosticada e tratada oportunamente. Devido a sua transcendência e magnitude, configura-se como problema de saúde pública de relevância social e epidemiológica em vários países. No ano de 2018 foram notificados 208.619 casos novos da doença em 159 países. O Sudeste asiático e as Américas apresentaram as maiores taxas de detecção: 7,49 e 3,08 casos novos por 100 mil habitantes respectivamente. Os três países que concentram alta carga da doença são Índia, Brasil e Indonésia ${ }^{(1)}$.

A continuidade da transmissão da doença, além das causas biológicas, genética e imunológica $^{(2-3)}$, está relacionada aos múltiplos fatores de exposição, aos processos migratórios ${ }^{(4)}$, à inacessibilidade aos serviços básicos de saúde e condições socioeconômicas desfavoráveis ${ }^{(5-6)}$.

No Brasil, embora haja tendência decrescente da hanseníase em âmbito nacional, um estudo que analisou a doença no período de 2001 a 2015 revelou o comportamento focal e desigual entre as regiões do Brasil. As Regiões Norte, Centro-Oeste e Nordeste apresentam alta carga da doença ${ }^{(7)}$.

Na Região Nordeste, o estado da Paraíba apresenta tendência decrescente, no entanto, ainda se encontra em alta endemicidade de acordo com os parâmetros do Ministério da Saúde (MS). Ressalta-se que a transmissão continua ativa tendo em vista a incidência de casos na população adulta e em menores de 15 anos, fato que pressupõe a presença de adultos doentes sem diagnóstico e/ou sem tratamento ${ }^{(8-9)}$. Além disso, são notificados casos com grau 2 de incapacidade física, isto é, com deformidades e incapacidades instaladas, fato que prediz diagnóstico tardio, aponta baixa eficiência de detecção precoce pelos profissionais de saúde e contribui para prevalência oculta (casos não diagnosticados) ${ }^{(8,10)}$.

No Brasil, as ações de controle da hanseníase, a detecção oportuna de novos casos, o tratamento com o esquema poliquimioterápico, a vigilância dos contatos, a prevenção de incapacidades e a reabilitação são descentralizadas para a Atenção Primária à Saúde (APS), que está ancorada na Estratégia Saúde da Família (ESF), composta por uma equipe interdisciplinar ${ }^{(9)}$. O enfermeiro, como parte integrante da equipe, assume papel decisivo e proativo nas ações de controle da hanseníase, tais como: busca ativa de casos, vigilância de contatos, educação em saúde, prevenção e tratamento das incapacidades, vigilância epidemiológica, além de contribuir para pesquisas ${ }^{(11)}$.

As metas de eliminação são, proporcionalmente, mais viáveis quanto melhor a qualidade dos serviços de saúde. Nesse sentido, o estudo dos indicadores de monitoramento da doença tem sido utilizado pelos profissionais de saúde e gestão para acompanhar o progresso de eliminação da hanseníase como problema de saúde pública. A Organização Mundial da Saúde (OMS) recomenda o uso do geoprocessamento para analisar a distribuição espacial de áreas que ofereçam maior risco de adoecimento para subsidiar o planejamento das ações de saúde ${ }^{(12)}$. Destaca-se que são limitados os estudos que apontem áreas de risco para hanseníase, por meio da análise espacial no estado da Paraíba, Brasil. 
Assim, a realização deste estudo justifica-se pela relevância em conhecer a distribuição da hanseníase em diferentes estados brasileiros, bem como a formação de áreas de risco que mantém a continuidade da cadeia de transmissão. Ainda, pela contribuição dos resultados para os profissionais de saúde e gestores nas reflexões das práticas de vigilância epidemiológica e ações de atenção à hanseníase. Nesse sentido, o objetivo deste estudo é analisar a distribuição espacial do risco para o adoecimento de hanseníase nos municípios do estado da Paraíba, Brasil.

\section{Método}

Trata-se de estudo ecológico com análise espacial. Utilizou-se como unidade de análise os 223 municípios do estado da Paraíba, que conta com uma população estimada de 4.018.127 habitantes ${ }^{(13)}$.

A população foi composta por casos novos de hanseníase residentes na Paraíba, registrados no Sistema de Informação de Agravos de Notificação (SINAN), no período de 2001 a 2016, disponibilizados pela Secretaria Estadual de Saúde da Paraíba.

As estimativas anuais da população geral e categorizadas por idade ( $<15$ anos), utilizadas para calcular os indicadores, foram obtidas pelo Instituto Brasileiro de Geografia e Estatística (IBGE) e o Departamento de Informática do Sistema Único de Saúde, respectivamente ${ }^{(14)}$.

Dividiu-se o tempo em dois períodos (2001 a 2008 e 2009 a 2016). Essa divisão teve como intuito avaliar as oscilações dos indicadores, mediante a implantação de políticas de saúde, sociais e estratégias de mobilização e intensificação das ações para o controle da hanseníase nos municípios.

O processamento e análise dos dados ocorreram entre os meses de fevereiro e junho de 2018. Foi realizada análise de consistência por meio do Office Excel 2010. Entre os 13.603 casos de hanseníase registrados, foram incluídos no estudo os casos novos como modo de entrada e excluídos os erros diagnósticos, os casos de residentes em outros estados ou países e aqueles notificados fora do período de estudo, totalizando 12.134 casos novos de hanseníase.
Calcularam-se os indicadores de avaliação e monitoramento da hanseníase preconizados pelo MS: taxa de detecção de casos novos de hanseníase na população geral/100 mil habitantes (mede a força da morbidade, magnitude e tendência da endemia); taxa de detecção de casos novos de hanseníase em menores de 15 anos/100 mil habitantes (mede a força da transmissão recente e tendência da endemia); taxa de casos novos de hanseníase com grau 2 de incapacidade física no momento do diagnóstico/100 mil habitantes (avalia as deformidades causadas pela hanseníase na população e monitora a tendência de detecção oportuna dos casos novos de hanseníase) $)^{(9)}$.

Após a integralização desses parâmetros foi construído um indicador composto denominado "Índice de risco de adoecimento da hanseníase". A escolha dessa metodologia justifica-se por permitir avaliar o risco de adoecimento de forma ampliada, não se detendo apenas na análise de um indicador isolado.

A integralização dos indicadores foi realizada da seguinte forma: calculou-se por município a média dos indicadores epidemiológicos para os períodos 2001 a 2008 e 2009 a 2016, e aplicou-se equação de transformação em scores para cada indicador, por meio da razão: = [(valor observado/ valor máximo)] = scores dos indicadores

Em seguida, os scores dos indicadores foram somados e divididos por três, e os valores transformados em índices: Índice $=$ Soma dos scores dos indicadores de hanseníase dividido pelo número de indicadores. Os valores do índice variaram entre 0 e 1 , considerando "melhor" o de menor valor (0), e "pior", o de maior valor (1).

A apresentação espacial do Índice de risco de adoecimento de hanseníase foi realizada mediante quartis de distribuições. No primeiro período (2001 a 2008) os valores foram classificados em muito baixo risco $(<0,02)$; baixo risco $(0,02 \mid--0,04)$; médio risco $(0,04 \mid--0,12)$; e alto risco $(>0,12)$. Para o segundo período (2009 a 2016) os valores foram categorizados em muito baixo risco $(<0,02)$; baixo risco $(0,02 \mid--0,07)$; médio risco $(0,07 \mid--0,15)$; e alto risco $(>0,15)$.

A fim de verificar a autocorrelação espacial global, utilizou-se como ferramenta estatística o 
Índice de Moran global e local Local Index of Spatial Association (LISA) por meio do Índice de Moran local, que permite verificar o quanto o valor analisado do índice em um determinado município é dependente de valores avaliados desse mesmo índice em municípios vizinhos ${ }^{(15)}$.

Os resultados do Índice de risco de adoecimento de hanseníase estão apresentados em mapas coropléticos (temáticos) que representam os dados de uma área em cores. No tocante à apresentação dos resultados do Índice local de Moran, optou-se pelo Box Map, no qual cada município é classificado conforme sua posição em relação aos quadrantes do gráfico de espalhamento de Moran.

Os quadrantes gerados nessa técnica são interpretados da seguinte forma: Q1 - alto/alto (valores positivos, médias positivas) mostra municípios com altos índices, cercados de outros municípios também com altos índices; Q2 - baixo/baixo (valores negativos, médias negativas): municípios com baixo valor do índice, cercados por municípios com baixo valor do mesmo índice; Q3 - alto-baixo (valores positivos, médias negativas): municípios com valores altos do índice, cercados por municípios com baixos valores desse menos índice; Q4 - baixo-alto (valores negativos, médias positivas): municípios com valores do índice baixos, cercados de municípios com valores altos do mesmo índice.
O estudo foi aprovado pelo Comitê de Ética em Pesquisa (CEP) da Universidade Federal de Minas Gerais, CAAE 62339616.9.0000.5149, sob Parecer $n^{\mathrm{O}}$ 1.873.644.

\section{Resultados}

Foram registrados 12.134 casos novos de hanseníase no período estudado. Entre os anos de 2001 e 2008, a taxa de detecção geral da hanseníase teve média de 15,31 casos/100 mil habitantes. Entretanto, em menores de 15 anos, a média foi de 3,23 casos/100 mil habitantes e a taxa de grau 2 de incapacidade, de 0,99/100 mil habitantes.

Considerando o período de 2009 a 2016, houve diminuição nos indicadores da hanseníase: a média da taxa de detecção geral passou para 11,79/100 mil habitantes; em menores de 15 anos, para 2,34/100 mil; e a taxa de grau 2 de incapacidade física, para 0,84/100 mil habitantes.

A Figura 1 apresenta o mapa do índice de risco de adoecimento da hanseníase, no período de 2001 a 2008. Observa-se que 56 (25\%) municípios estavam classificados em muito baixo risco para adoecimento pela hanseníase, 48 (22\%), em baixo; 63 (28\%), em médio; e 56 (25\%), em alto risco. Os municípios classificados em alto risco localizavam-se principalmente nas regiões leste, sudeste, oeste e noroeste do estado.

Figura 1 - Mapa da distribuição espacial do índice de risco de adoecimento da hanseníase. Paraíba, Brasil - 2001-2008

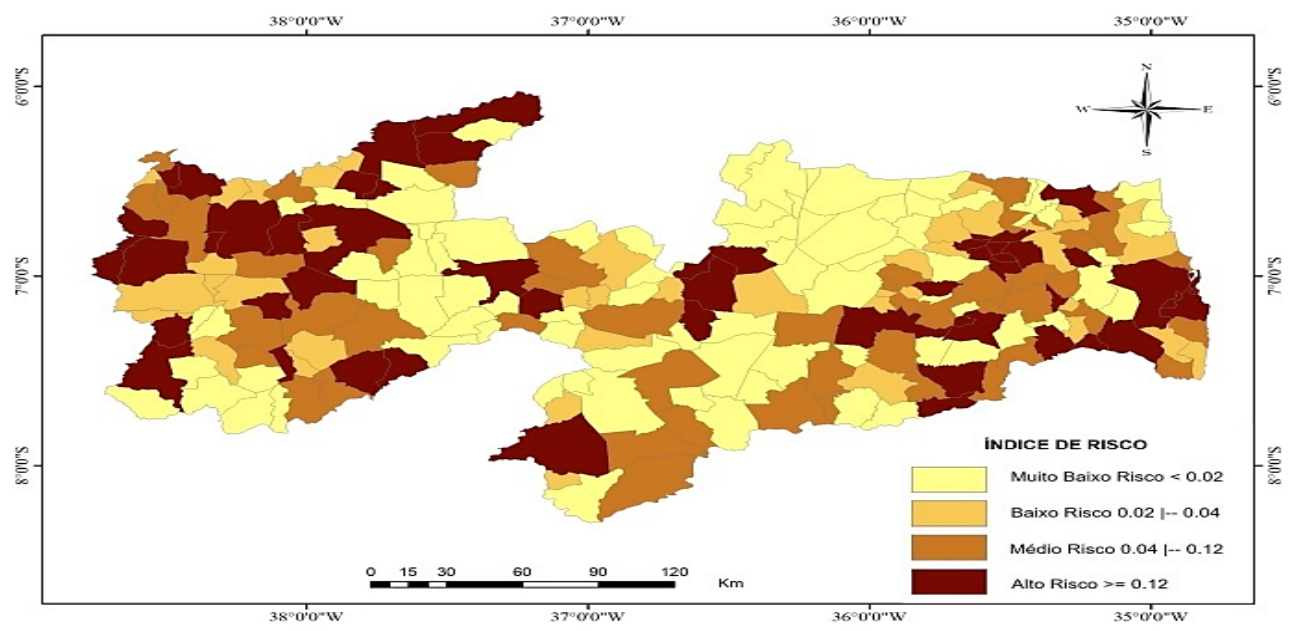

Fonte: Elaboração própria. 
O Índice Global de Moran confirmou a existência de dependência espacial entre os municípios $(0,21 ; p=0,004)$. Assim, quando realizado o Índice Local de Moran, observou-se a existência de clusters em municípios de baixo risco localizados nas regiões centro-norte do estado, principal aglomerado na divisa com o estado do Rio Grande do Norte, e aglomerados de alto risco nas regiões leste, oeste e noroeste (Figura 2).

Figura 2 - Mapa da estatística espacial do índice de risco de adoecimento por hanseníase. Paraíba, Brasil - 2001-2008

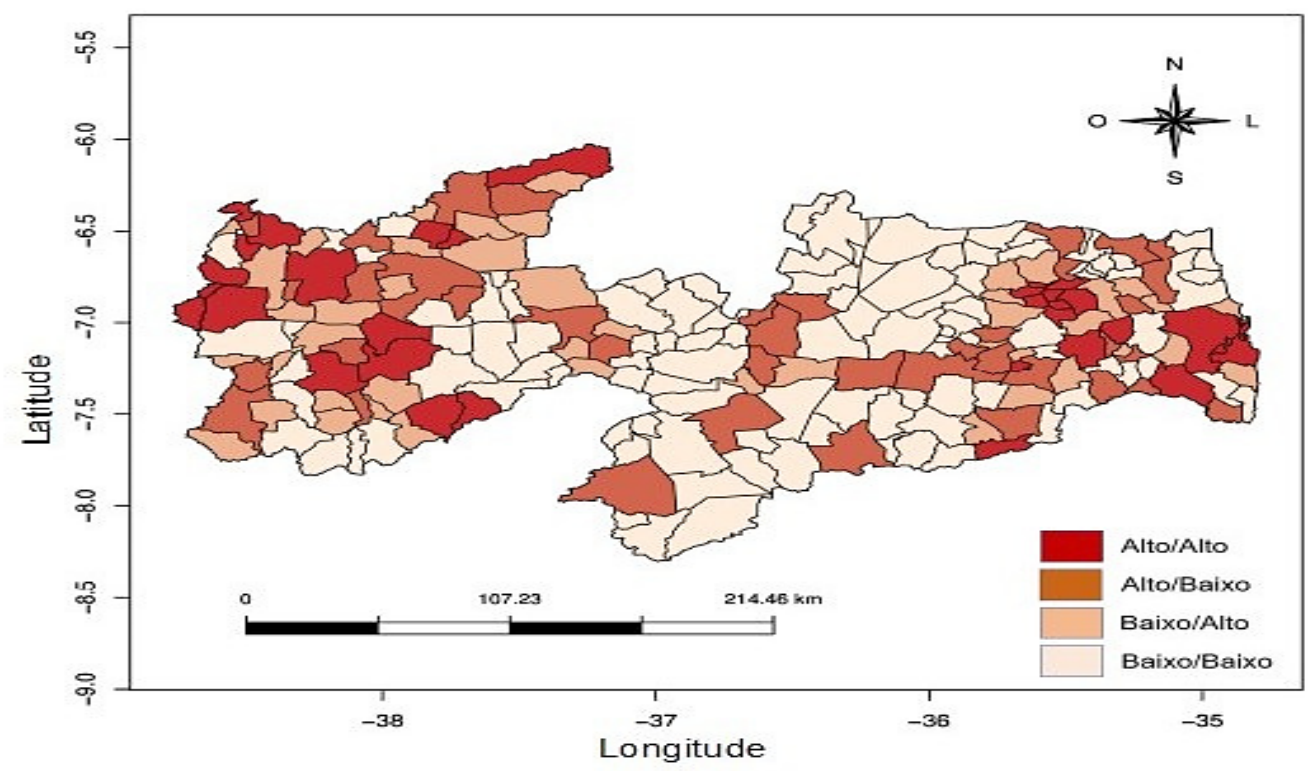

Fonte: Elaboração própria.

Apresentaram significância estatísticas na região oeste (Região Intermediária de Cajazeiras-Sousa), os municípios de Cajazeiras, Cachoeira dos Índios, Bom Jesus, Santa Helena e Sousa. Ainda, os municípios de Coremas, Piancó e Itaporanga (Região Intermediária de Patos). Na região leste (Região Intermediária de João Pessoa), João Pessoa, Santa Rita, Bayeux, Alagoinha, Cuitegi, Guarabira, Pilões, Borborema, e no sudeste, o município de Pedras de Fogo.
Para o período de 2009 a 2016, observa-se que os municípios classificados em muito baixo risco diminuíram para 47 (21\%); o baixo risco aumentou para 64 (29\%); o médio risco diminuiu para 54 (24\%) e o alto risco aumentou para dois, totalizando 58 (26\%). Os municípios de alto risco permaneceram em maior quantidade concentrados nas regiões oeste, leste e sudeste do estado (Figura 3). 
Figura 3 - Mapa da distribuição espacial do índice de risco de adoecimento da hanseníase. Paraíba, Brasil - 2009-2016

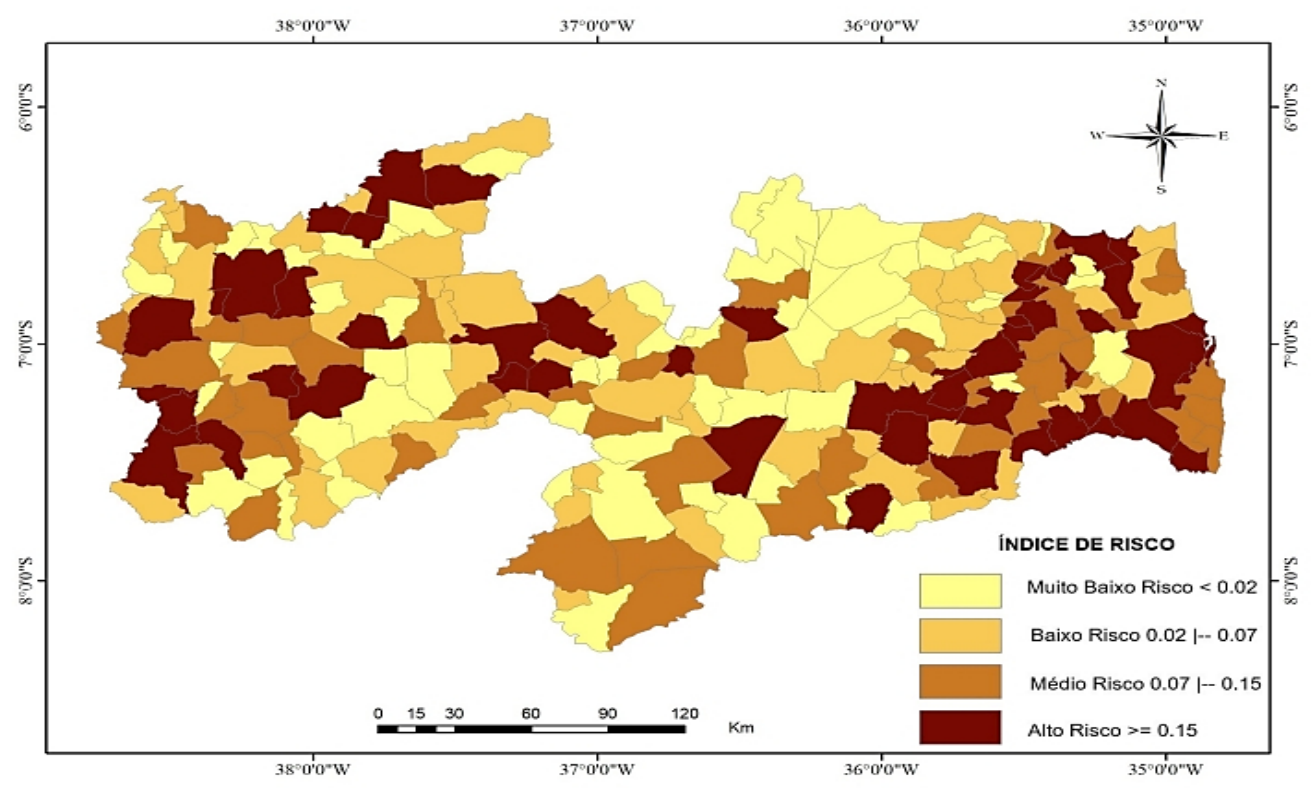

Fonte: Elaboração própria.

O Índice Global de Moran identificou dependência espacial entre as áreas da região $(0,22$; $\mathrm{p}<0,03)$, o que permitiu a aplicação do Índice de Moran Local. Observa-se nesse período, no Box Map, aumento do cluster de baixo risco no norte/nordeste do estado, comparado ao período anterior, e aumento dos clusters de municípios de alto risco localizados na região leste e sudeste e redução no oeste e noroeste (Figura 4).

Figura 4 - Mapa da estatística espacial do índice de risco de adoecimento da hanseníase. Paraíba, Brasil $-2009-2016$

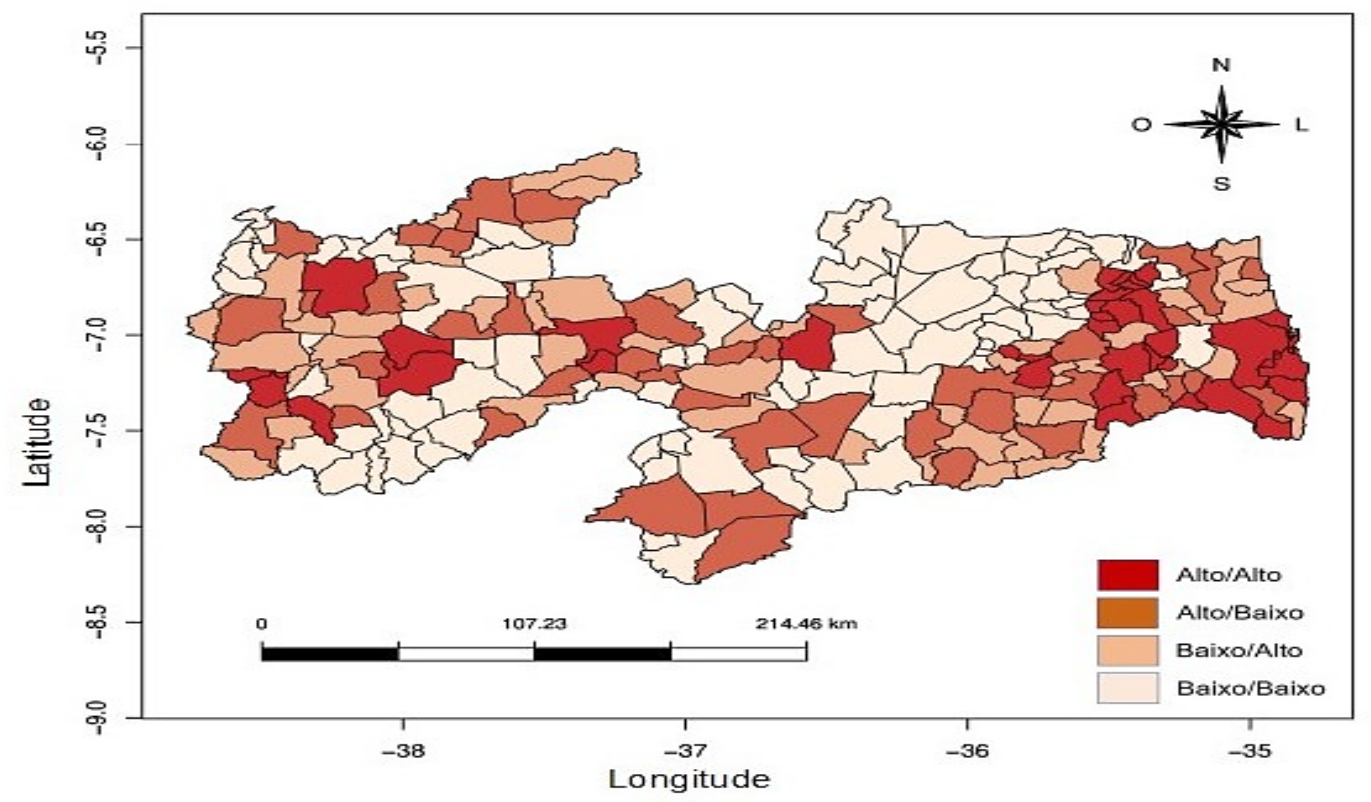

Fonte: Elaboração própria. 
Apresentou significância estatística ao oeste (Região Intermediária Cajazeiras-Sousa) apenas o município de Sousa, além de permanecer Coremas e Piancó (Região Intermediária de Patos).

Na região leste (Região Intermediária de João Pessoa), permaneceram Bayeux, Alagoinha, Cuitegi, Guarabira, com incremento de Lucena. No Sudeste permaneceu Pedras de Fogo, com acréscimo do município de Juripiranga.

\section{Discussão}

Neste estudo, os coeficientes de detecção da hanseníase apontaram para persistência da doença e situação de alta endemicidade no estado da Paraíba. Municípios classificados em alto risco estavam distribuídos em todo o estado, entretanto, a maior concentração localizou-se nas regiões noroeste e oeste, fronteira com o estado do Ceará, e nas regiões leste e sudeste, limites contínuos com o estado de Pernambuco, formando assim, chusters nas regiões leste e oeste do estado.

Os estados do Ceará e Pernambuco apresentam altas taxas de detecção da hanseníase ${ }^{(16)}$ e estão classificados em "muito alta endemicidade"(9). Assim, a vizinhança da Paraíba com esses estados pode, por meio das migrações interestaduais, reforçar a transmissibilidade da doença. Estudo realizado na fronteira tríplice da América Latina (Brasil, Argentina e Paraguai) aponta para grande fluxo de pessoas entre os três países, o que torna o controle de doenças transmissíveis altamente complexo ${ }^{(17)}$.

Na Grã-Bretanha e outros países europeus, embora a doença tenha sido eliminada, está confinada aos imigrantes de países endêmicos. Na Dinamarca, Canadá e Espanha foi comprovado que o pequeno número de casos registrados em dez anos foi de imigrantes de países endê$\operatorname{micos}^{(18-19)}$. Assim, mesmo em países que não tenha casos da doença, o sistema de vigilância deve estar ativo e com profissionais de saúde capacitados para reconhecer os sinais e sintomas.

$\mathrm{Na}$ região leste, o aglomerado de municípios de alto risco pertence em parte à região metropolitana da capital do estado, João Pessoa. Essa região tem alta densidade demográfica, elevada circulação de pessoas, comércio e prestação de serviços e melhor Índice de Desenvolvimento Humano Municipal (IDHM) ${ }^{(13)}$. Esse fato pode ter relação com melhor acesso aos serviços de referência, e assim melhor capacidade para o diagnóstico.

Estudo realizado no estado do Tocantins, Brasil constatou que a detecção da doença foi significativamente maior em municípios com melhor IDHM, entretanto, esse fato foi explicado pela abrangência de áreas de maior densidade demográfica, maior circulação de pessoas e desenvolvimento ${ }^{(20)}$.

No Brasil, a hanseníase em áreas urbanas tem sido explicada devido à rápida urbanização e migração populacional intensa ${ }^{(4)}$. Tal fato acarreta maior densidade populacional em determinadas áreas, crescimento desorganizado e contribui para fomentar as desigualdades sociais, excluindo as pessoas de oportunidades sociais e materiais de sobrevivência, bem como do acesso aos serviços de saúde.

Na região oeste da Paraíba, alguns municípios encontrados em alto risco, embora tenham população abaixo de 60 mil habitantes, são localidades que apresentam histórico anterior de hiperendemicidade de hanseníase, a exemplo dos municípios Cajazeiras, Conceição, Catolé do Rocha e Sousa, apontados nos clusters em estudo realizado no Brasil, no período de 2005 a $2007^{(21)}$, e Cuitegi, presente no cluster de alto risco de detecção de hanseníase no Brasil, no período de 2001 a $2015^{(7)}$.

Embora a hanseníase apresente declínio na Paraíba ${ }^{(8)}$ e tenha ocorrido diminuição dos municípios de alto risco na região oeste do estado, aumentou a concentração dos municípios classificados em alto risco nas regiões leste e sudeste. Tal fato reforça a ideia de persistência temporal e geográfica da doença. A concentração espacial da hanseníase e a persistência de determinadas áreas geográficas têm sido mostradas em estudos $^{(7,20)}$.

A persistência na análise espacial no segundo período de estudo dos municípios na região leste, área metropolitana de João Pessoa, 
particularmente Bayeux, pode estar associada, além da densidade populacional, a determinantes histórico-sociais, posto que, no passado, nesse município funcionava o antigo leprosário da Paraíba, Hospital Colônia Getúlio Vargas. Dessa forma, observa-se um determinante histórico-social que marca o presente e que os serviços de saúde não conseguem romper.

De fato, áreas com histórico de endemicidade pode haver uma prevalência oculta que contribua com a cadeia de transmissão da doença. No distrito de Munger, estado indiano de Bihar, considerado endêmico para hanseníase, após atividade intensiva de busca ativa de casos, no ano de 2016 foram diagnosticados 321 novos casos da doença entre 85.560 pessoas examinadas ${ }^{(22)}$.

A explicação para alguns municípios aumentarem ou permanecerem em alto risco entre os períodos analisados, bem como aumento de clusters de alto risco, pode estar associada a alguns fatores: longo período de incubação do Mycobacterium leprae, qualidade dos serviços de saúde, condições socioeconômicas desfavoráveis presentes em determinadas áreas municipais e qualidade da informação em saúde ${ }^{(6,23)}$.

Há evidências de que o comportamento epidemiológico da hanseníase é influenciado pela qualidade e capacidade operacional dos serviços de saúde da APS, sendo mais efetivos aqueles serviços que detectam e tratam precocemente os casos, prevenindo a ocorrência de incapacidades físicas e estão atentos às necessidades do usuário, desde a prevenção de agravos até a reabilitação ${ }^{(24)}$. Na Etiópia, as ações de controle da hanseníase foram descentralizadas para serviços básicos de saúde, com o intuito de aproximar a população do diagnóstico precoce, tratamento oportuno e acompanhamento dos casos, e apresentou melhoria nos indicadores da doença ${ }^{(25)}$.

No entanto, quando as ações de controle da hanseníase não são desenvolvidas com qualidade, podem, por vezes, explicar a persistência do risco do adoecimento por hanseníase em determinadas áreas geográficas. Por isso, os serviços devem estar providos de recursos humanos capacitados para acolher o usuário e desenvolver as ações de controle da hanseníase de forma adequada ${ }^{(11)}$.

Dessa forma, destaca-se a importância do trabalho do enfermeiro nas ações de vigilância da hanseníase, uma vez que esse profissional assume diversas funções de planejamento, execução e avaliação das atividades de controle, gestão, vigilância epidemiológica, além de estar em articulação com outros profissionais para garantir o diagnóstico precoce da doença, o tratamento, e o acompanhamento do usuário e família ${ }^{(11)}$.

Portanto, torna-se necessário iniciar a aproximação com a hanseníase no processo de formação nas diversas áreas de saúde, especialmente para aqueles profissionais que irão atuar no futuro nos serviços de APS. Além disso, desenvolver atividades de educação permanente em saúde para profissionais da ESF e evitar a rotatividade de profissionais são medidas que podem contribuir para o controle da doença.

Adicionalmente, vulnerabilidades sociais e iniquidades presentes em determinadas áreas colocam em risco a saúde da população. Estudos espaciais mostram que a distribuição da doença no espaço é influenciada pelas desigualdades, condições socioeconômicas desfavoráveis e fatores culturais ${ }^{(23)}$. As ações a serem desenvolvidas para melhor controle da doença não dependem apenas dos serviços de saúde, a ênfase nas ações de combate à pobreza, melhorias educacionais, geração de emprego e renda, além de promoverem o acesso aos serviços de saúde, são efetivas no controle da doença.

Os municípios que foram classificados em baixo risco formaram clusters no primeiro período na região centro-norte, e no segundo período, no norte do estado. Estes devem ser destacados e analisados com cautela referente ao adoecimento pela hanseníase. Isso porque esses municípios podem realmente estar livres da doença, mas, por outro lado, é possível a ocorrência de erros diagnósticos, subnotificações e/ou diagnóstico tardio ${ }^{(19)}$.

Os municípios com muito baixo risco, fazendo fronteira com municípios que apresentam alto risco, podem também influenciar a cadeia 
de transmissão pela circulação de pessoas. Dessa forma, serve como alerta de vigilância a necessidade de intensificar ações de busca ativa para detectar casos de difícil diagnóstico que possam contribuir no futuro próximo para a persistência da hanseníase.

Este estudo tem limitações, uma vez que os dados utilizados são de fonte secundária, que podem apresentar inconsistência na qualidade e quantidade das informações. Apesar disso, os dados foram tratados com rigor metodológico e são representativos da situação epidemiológica da hanseníase no período estudado.

\section{Conclusão}

Em conclusão, a análise espacial contribuiu para identificar áreas focais da hanseníase no estado da Paraíba. A distribuição da doença é dessemelhante no estado, e clusters de alto risco estão presentes nas regiões leste e oeste.

Essas áreas podem ser compensadas pela organização e estruturação dos serviços de saúde, garantia do acesso equânime da população e a integralidade das ações para o controle da endemia. Ressalta-se que novas abordagens de pesquisa devem acontecer para melhor conhecimento dos fatores condicionantes e determinantes da doença.

Os resultados deste estudo contribuem para promover reflexões entre profissionais de saúde e gestores sobre as ações de controle da hanseníase, além de contribuir para a comunidade científica, o processo de formação em saúde visando direcionar ações para o controle da doença. Além disso, exige novas abordagens de pesquisa para melhor compreender fatores que condicionam e/ou determinam a manutenção da doença em diversos municípios do estado.

\section{Colaborações:}

1 - concepção, projeto, análise e interpretação dos dados: Kleane Maria da Fonseca Azevedo Araújo, Lidiane Cristina Félix Gomes e Francisco Carlos Félix Lana;
2 - redação do artigo e revisão crítica relevante do conteúdo intelectual: Kleane Maria da Fonseca Azevedo Araújo e Francisco Carlos Félix Lana;

3 - aprovação final da versão a ser publicada: Kleane Maria da Fonseca Azevedo Araújo, Lidiane Cristina Félix Gomes e Francisco Carlos Félix Lana.

\section{Referências}

1. World Health Organization. Global leprosy update, 2018: moving towards a leprosy-free world. Weekly Epidemiol Rec [Internet]. 2019 [cited 2019 Dec 10]; 94(35-36):389-412. Available from: https:// apps.who.int/iris/bitstream/handle/10665/326775/ WER9435-36-en-fr.pdf?ua=1

2. Masaki T, McGlinchey A, Cholewa-Waclaw J, Qu J, Tomlinson SR, Rambukkana A. Innate Immune Response Precedes Mycobacterium Leprae-Induced Reprogramming of Adult Schwann Cells. Cell Reprogram. 2014;16(1):9-17. DOI: $10.1089 /$ cell.2013.0064

3. Benjak A, Avanzi C, Singh P, Loiseau C, Girma S, Busso P, et al. Phylogenomics and Antimicrobial Resistance of the Leprosy Bacillus Mycobacterium Leprae. Nat Commun. 2018;9(1):352-62. DOI: 10.1038/s41467-017-02576-Z

4. Murto C, Ariza L, Alencar CH, Chichava OA, Oliveira AR, Kaplan C, et al. Migration among individuals with leprosy: a population-based study in Central Brazil. Cad Saúde Pública. 2014;30(3):487-501. DOI:10.1590/0102311X00005913

5. Nery JS, Ramond A, Pescarini JM, Alves A, Strina A, Ichiara MY, et al. Socioeconomic determinants of leprosy new case detection in the 100 Million Brazilian Cohort: a population-based linkage study. The Lancet. 2019;7:30260-8. DOI:10.1016/S2214-109X(19)30260-8

6. Leano HAM, Araújo KMFA, Bueno IC, Niitsuma ENA, Lana FCF. Fatores socioeconômicos relacionados à hanseníase: revisão integrativa da literatura. Rev Bras Enferm. 2019;72(5):1405-15. DOI: 10.1590/0034$7167-2017-0651$

7. Rodrigues RN, Leano HAM, Bueno IC, Araújo KMFA, Lana FCF. High-risk areas of leprosy in Brazil between 2001-2015. Rev 
Bras Enferm. 2020;73(3):e20180583. DOI: 10.1590/0034-7167-2018-0583

8. Araújo KMFA, Leano HAM, Rodrigues RN, Bueno IC, Lana FCF. Tendência de indicadores epidemiológicos da hanseníase em um estado endêmico. Rev Rene. 2017;18(6):771-8. DOI: 10.15253/2175-6783.2017000600010

9. Brasil. Ministério da Saúde. Secretaria de Vigilância em Saúde. Diretrizes para vigilância, atenção e eliminação da Hanseníase como problema de saúde pública: manual técnico-operacional [Internet]. Brasília (DF); 2016 [cited 2019 Dec 10]. Available from: http://www.saude.gov.br/images/ pdf/2016/fevereiro/04/diretrizes-eliminacaohanseniase-4fev16-web.pdf

10. Souza EA, Ferreira AF, Boigny RN, Alencar CH, Heukelbach J, Martins-Melo FR, et al. Leprosy and gender in Brazil: trends in an endemic area of the Northeast region, 2001-2014. Rev Saude Publica. 2018;52:20. DOI:10.11606/ s1518-8787.2018052000335

11. Sousa GS, Silva RLF, Xavier MB. Atributos da atenção primária em saúde no controle da hanseníase: ótica do enfermeiro. Rev baiana enferm. 2017;31(1):e17251. DOI: http://dx.doi. org/10.18471/rbe.v31i1.17251

12. Word Health Organization. Global leprosy update, 2016: accelerating reduction of disease burden. Weekly Epidemiol Rec [Internet]. 2017 [cited 2019 Dec 15];35(92):501-20. Available from: https:// apps.who.int/iris/bitstream/e/10665/258841/9235 pdf;jsessionid=A10D667A466A3DAB793B56498C6 7B373? sequence $=1$

13. Instituto Brasileiro de Geografia e Estatística. Cidades [Internet] Rio de Janeiro (RJ); 2019 [cited 2020 May 10]. Available from: https:// cidades.ibge.gov.br/brasil/pb/panorama

14. Brasil. Ministério da Saúde. Departamento de Informática do SUS-DATASUS. Informações de Saúde, Demográficas e Socioeconômicas [Internet] Brasília (DF); 2018 [cited 2018 Nov 20]. Available from: http://www.datasus.gov.br/DATASUS/index. php?area $=0206 \&$ id $=6942$

15. Souza WV, Carvalho MS, Cruz OG, Ragoni V. Análise espacial de dados de área. In: Santos SM, Souza WV, organizadores. Introdução à Estatística Espacial para a Saúde Pública. Brasília: Ministério da Saúde; 2007. p. 63-78.
16. Leano HAM, Araújo KMFA, Rodrigues RN, Bueno IC, Lana FCF. Indicadores relacionados a incapacidade física e diagnóstico de hanseníase. Rev Rene. 2017;18(6):832-9. DOI: $10.15253 / 2175-6783.2017000600018$

17. Assis IS, Arcoverde MAM, Ramos ACV, Alves LS, Berra TZ, Arroyo LH, et al. Social determinants, their relationship with leprosy risk and temporal trends in a tri-border region in Latin America. PLoS Negl Trop Dis. 2018;12(4):e0006407. DOI: 10.1371/ journal.pntd.0006407

18. Aftab H, Nielsen SD, Bygbjerg C. Leprosy in Denmark 1980-2010: a review of 15 cases. BMC Res Notes. 2016;9(10):2-9. DOI: 10.1186/ s13104-015-1768-6

19. Ramos JM, Romero D, Belinchón I. Epidemiology of Leprosy in Spain: The Role of the International Migration. PLoS NeglTrop Dis. 2016;10(3):e0004321. DOI: 10.1371/journal.pntd.0004321

20. Monteiro LD, Mota RMS, Martins-Melo FR, Alencar CH, Heukelbach J. Determinantes sociais da hanseníase em um estado hiperendêmico da região Norte do Brasil. Rev Saude Publica. 2017;51(70):1-11. DOI:10.1590/s15188787.2017051006655

21. Penna MLF, Oliveira MLV, Penna GO. The epidemiological behaviour of leprosy in Brazil. Lepr Rev [Internet]. 2009 [cited 2019 Dec 20];80(3):332-44. Available from: https://pdfs.semanticscholar.org/9a4c/ $4 \mathrm{c} 74 \mathrm{cc} 700 \mathrm{f} 0 \mathrm{c} 1 \mathrm{~b} 2 \mathrm{aac} 5 \mathrm{ebd} 87 \mathrm{ec} 0234443611$. pdf

22. Mangeard-Lourme J, Singh A, Singh RK, Parasa J, Arquer GR. Enhanced active case-finding, identifying leprosy cases missed by recent detection campaigns in Munger District, Bihar, India. Lepr Rev [Internet]. 2017 [cited 2019 Dec 10];88:452-62. Available from: https://pdfs.semanticscholar.org/ d8d7/523769d6e457e10aed6b7bdb1ee703250c50. pdf?_

23. Rodrigues RN, Niitsuma ENA, Bueno IC, Baquero OS, Jardim CCG, Lana FCF. Hanseníase e vulnerabilidade da saúde em Belo Horizonte, Minas Gerais. REME rev min enferm. 2017;21:e-997. DOI:10.5935/1415-2762.20170007

24. Lanza FM, Lana FCF. O processo de trabalho em hanseníase: tecnologias e atuação da equipe de saúde da família. Texto contexto enferm. 2011;20(spe):238-46. DOI:10.1590/S010407072011000500030 
Mailson Marques de Sousa, Rhaira Pereira Campos, Jacira dos Santos Oliveira, Simone Helena dos Santos Oliveira

25. Abeje T, Negera E, Kebede E, Hailu T, Hassen I, Lema $\mathrm{T}$, et al. A. Performance of general health workers in leprosy control activities at public health facilities in Amhara and Oromia States, Ethiopia. BMC Health Serv Res. 2016;7(16):122. DOI: 10.1186/s12913-016-1329-2
Recebido: 15 de julho de 2020 Aprovado: 26 de agosto de 2020

Publicado: 7 de outubro de 2020

A Revista Baiana de Enfermagem utiliza a Licença Creative Commons - Atribuição-NãoComercial 4.0 Internacional. https://creativecommons.org/licenses/by-nc/4.0/ Este artigo é de acesso aberto distribuído sob os termos da Licença Creative Commons (CC BY-NC). Esta licença permite que outros remixem, adaptem e criem a partir do seu trabalho para fins não comerciais. Embora os novos trabalhos tenham de lhe atribuir o devido crédito e não possam ser usados para fins comerciais, os usuários não têm de licenciar esses trabalhos derivados sob os mesmos termos. 